\title{
A Density Functional Theory- and Atoms in Molecules-based Study of NiNTA and NiNTPA Complexes toward Physical Properties Controlling their Stability. A New Method of Computing a Formation Constant.
}

\author{
Ignacy Cukrowski* and Krishna K. Govender
}

Department of Chemistry, Faculty of Natural Sciences, University of Pretoria, Lynnwood Road, Hillcrest, Pretoria 0002, South Africa

Email: ignacy.cukrowski@up.ac.za

Keyword: DFT; QTAIM, Formation constant, Nickel, Carboxylic acids, Chelating effect, Strain energy, $\mathrm{H}-\mathrm{H}$ bond, $\mathrm{CH}-\mathrm{O}$ bond. 


\begin{abstract}
The $\log K_{1}$ value of analytical quality was obtained for the NiNTPA complex using the DFTcomputed (at the B3LYP/6-311++G(d,p) level of theory in solvent, CPCM/UAKS) G(aq) values of the lowest energy conformers of the ligands, NTA (nitrilotriacetic acid) and NTPA (nitrilotri-3-propanoic acid), and Ni(II) complexes (NiNTA and NiNTPA). The described mathematical protocol is of general nature. The topological analysis, based on the Quantum Theory of Atoms in Molecules (QTAIM) of Bader, was used to characterize coordination bonds, chelating rings and additional intramolecular interactions in the complexes. The topological data, but not the structural analysis, explained the observed difference in stability of the NiNTA and NiNTPA complexes. It was found that the structural $\mathrm{H} \cdots \mathrm{H}$ contacts (classically regarded $\mathrm{H}$-clashes, a steric hindrance destabilizing the complex) are in fact the $\mathrm{H}-$ $\mathrm{H}$ bonds contributing to the overall stability of NiNTPA. Also a $\mathrm{CH}-\mathrm{O}$ bond was found in NiNTPA. The absence of intramolecular bonds between the atoms that fulfill a distance criterion in NiNTPA is explained by the formation of adjacent intramolecular rings that have larger electron density at the ring critical points when compared with the rings containing these atoms. It is postulated that the strength of a chelating ring (a chelating effect) can be measured by the electron density at the ring critical point. It was found that the strain energy, $E_{\mathrm{s}}$, in the as-in-complex NTPA ligand $\left(E_{\mathrm{s}}\right.$ is significantly lowered by the presence of the intramolecular bonded interactions found by QTAIM) is responsible for the decrease in strength of NiNTPA; the $E_{\mathrm{s}}$ ratio (NTPA/NTA) of 1.9 correlates well with the experimental $\log K_{1}$ ratio (NTA/NTPA) of 1.98.
\end{abstract}




\section{Introduction}

Knowledge of thermodynamic constants, such as protonation (or dissociation) constants of ligands and formation (stability) constants of metal complexes is of fundamental significance as they contribute toward understanding of the chemistry of metal ions with different ligands. They are important in many fields because their knowledge allows theoretical modeling of different media, such as natural waters (environmental applications), industrial solutions and effluents, or blood plasma (biomedical applications). ${ }^{1}$ Vigorous experimental investigation of protonation and formation constants has been conducted by different analytical techniques for many decades and dedicated compilations of reported data are available. ${ }^{2,3}$ Experimental data do not, however, provide sufficient theoretical insight on physical parameters controlling the affinity between metal ions and donor atoms as well as physical properties (on a fundamental level) of compounds (either ligands or metal complexes). Because of that, theoretical prediction of dissociation constants by use of computational techniques is being explored recently extensively ${ }^{4-11}$ but this does not apply to the formation constants of metal complexes yet. The use of density functional theory (DFT) to calculate the free energies, $G(\mathrm{~g})$, of components involved in a reaction of metal ions $\left(\left[\mathrm{M}\left(\mathrm{H}_{2} \mathrm{O}\right)_{6}\right]^{\mathrm{n}+}\right)$ in the gas phase with a single $\mathrm{NH}_{3}$ ligand (formation of an $\mathrm{ML}$ complex, $\left[\mathrm{M}\left(\mathrm{H}_{2} \mathrm{O}\right)_{5} \mathrm{NH}_{3}\right]^{\mathrm{n}+}$ ) has been reported recently. ${ }^{12,13}$ The DFT-computed $\Delta G(\mathrm{~g})$ values for a number of complex formation reactions, $\left(\left[\mathrm{M}\left(\mathrm{H}_{2} \mathrm{O}\right)_{6}\right]^{2+}\right.$ $+\mathrm{NH}_{3}=\left[\mathrm{M}\left(\mathrm{H}_{2} \mathrm{O}\right)_{5} \mathrm{NH}_{3}\right]^{2+}+\mathrm{H}_{2} \mathrm{O}$, have been used to generate the LFER (linear free-energy relationship) for divalent metal ions involving reported experimental values of $\Delta G(\mathrm{aq})$ for the relevant reaction in aqueous solution (or, equivalently, the $\log K_{1}$ value). A reasonable linear relationship was found but in some cases points were about $3 \mathrm{kcal} \mathrm{mol}^{-1}$ out of the fitted linear curve which is equivalent to about two log unit error in the predicted formation constant in solvent. Also, a LFER correlation between the DFT-calculated gas-phase proton macroaffinities (first protonation step) of metal complexes with the tripodal tetraamine ligands ${ }^{14}$ and triazacycloalkanes ${ }^{15}$ and corresponding formation constants in solution $\left(\log K_{1}\right)$ has been reported. The above methodologies involved gas phase calculations used to generate LFER from which $\log K_{1}$ values in solvent could be predicted with reasonable accuracy. However, these methodologies do not involve solvent-optimized structures hence no knowledge could be gained on physical properties of complexes formed. 
Interestingly, theoretical prediction (without a use of LFER) of formation constants in water, to the best of our knowledge, has not yet been reported even though one would expect that theoretical studies of metal complexes, when combined with reported formation constants, should explain why a small change in the ligand structure dramatically changes strength of complexes formed. For instance, it is commonly accepted that a six-membered chelating ring $(6 \mathrm{~m}-\mathrm{CR})$ is preferred by small metal ions and they form stronger complexes when compared with a similar ligand that forms a five-membered chelating ring (5m-CR). ${ }^{1,16,17}$ As a typical example one can consider ML complexes of small Be(II) (ionic radius $0.27 \AA)^{18}$ with NTPA (nitrilotri-3-propanoic acid) that has three $6 \mathrm{~m}$-CRs $\left(\log K_{1}=9.23 \text { at } 25^{\circ} \mathrm{C}, \mu=0.5 \mathrm{M} \mathrm{NaNO}_{3}\right)^{2}$ and NTA (nitrilotriacetic acid) that forms three $5 \mathrm{~m}-\mathrm{CR}\left(\log K_{1}=7.79\right.$ at $25^{\circ} \mathrm{C}, \mu=0.1 \mathrm{M}$ $\left.\mathrm{KNO}_{3}\right),{ }^{2} \Delta \log K_{1} \approx 1.4$ in favor of the 6-membered-ring structure. $\mathrm{Cd}(\mathrm{II})$ can be regarded as a large metal ion (ionic radius $0.95 \AA)^{18}$ and $\log K_{1}$ values of its ML complexes with NTPA and NTA are 3.4 (at $\left.30{ }^{\circ} \mathrm{C}, \mu=0.1 \mathrm{M} \mathrm{KNO}_{3}\right)^{2}$ and $9.76\left(\right.$ at $25^{\circ} \mathrm{C}, \mu=0.1 \mathrm{M} \mathrm{KNO}_{3}$ ), ${ }^{2}$ respectively, $\Delta \log K_{1} \approx 6.4$ in favor of the 5 -membered-ring structure. This kind of large variations in formation constants is often attributed to geometrical properties (bite angles and bond lengths) ${ }^{1,17}$ and crowded atoms in case of NTPA complexes with large metal ions. So-called $\mathrm{H}$-clashes are generally regarded as responsible for the large decrease in formation constants of a number of complexes. ${ }^{1,19-21}$

Our focus here is on $\mathrm{Ni}(\mathrm{II})$ (an average-size metal ion, ionic radius $0.69 \AA)^{18}$ complexes with NTA and NTPA where a decrease in formation constant of $\Delta \log K_{1} \approx 5.7$ is observed when three $5 \mathrm{~m}$-CRs present in NiNTA are replaced by three $6 \mathrm{~m}-\mathrm{CRs}$ to form NiNTPA. A competition reaction between NTA and NTPA for Ni(II) in the solvent (water) is used to theoretically compute a formation constant of the NiNTPA complex. The quantum theory of atoms in molecules (QTAIM) of Bader $^{22}$ provides a great deal of information about the nature of the bonding environment. We have explored the topological properties of the electron charge density, $\rho(\mathrm{r})$, and the Laplacian of the charge density, $\nabla^{2} \rho(\mathrm{r})$, at various bond critical points (BCPs) and ring critical points (RCPs) in the solvent-optimized conformers of NiNTA and NiNTPA to gain an insight on the physical properties of these complexes on a fundamental level (such as strength of coordination bonding, chelating effect, additional intramolecular interactions) with a hope to rationalize the observed change in the formation constants. 


\section{Computational methods}

Molecular modeling was performed with the aid of Gaussian 03, revision D01, software package. $^{23}$ GaussView 4.1.2 ${ }^{24}$ was utilized for molecular visualization and construction purposes. Constructed in GaussView molecules were finally optimized at the B3LYP/6$311++\mathrm{G}(\mathrm{d}, \mathrm{p})$ level of theory; since all molecules analyzed possessed negative charges it was essential that diffuse functions be included. ${ }^{25}$ Optimization of $\mathrm{Ni}$ (II) complexes was performed with a triplet spin multiplicity (octahedral complexes of nickel are considered here) as optimization using singlet spin multiplicity is used for $\mathrm{Ni}^{\mathrm{II}}$ complexes possessing square-planar geometry. ${ }^{26}$ Since the ultimate aim of our calculations is to rationalize and to predict formation constants of nickel complexes in aqueous solution, we therefore investigated the structural and electronic properties of each complex in solvent. For this purpose we have used the selfconsistent reaction field (SCRF) technique ${ }^{27-30}$ in which the statistically averaged effect of the solvent is simulated by representing the medium with appropriate physical properties, such as the dielectric permittivity $(\varepsilon)$ and the coefficient of thermal expansion. Dielectric continuum theories $^{31-34}$ are widely used to describe hydration because accurate results are produced at a relatively low computational cost; we have used the Conductor-like Polarizable Continuum Model (CPCM) $)^{35-39}$ in conjunction with the united atom (UA) cavity-model in-tagged with Kohn-Sham $(\mathrm{KS})$ radii $(\mathrm{UAKS})^{40-41}$ with water as a solvent $(\varepsilon=78.39)$. We performed frequency calculations by determining analytically the second derivatives of the UB3LYP potential energy surfaces with respect to the fixed atomic nuclear coordinates to determine whether each of the minimized structures corresponded to an energy minimum or a saddle point. A tight gradient convergence criterion (necessary for analyzing the small structural variations in these complexes) with ultrafine integration grid was used in all calculations. All reported geometries belong to genuine minimum energy conformations (imaginary frequencies are not present).

We carried out a topological analysis of the electron charge density $\rho(\mathrm{r})$ and the Laplacian of the charge density $\nabla^{2} \rho(\mathrm{r})$ at all critical points at the same level of theory (B3LYP/6$311++\mathrm{G}(\mathrm{d}, \mathrm{p}))$ using QTAIM of Bader. ${ }^{22}$ The AIMALL ${ }^{42}$ and AIM2000 ${ }^{43,44}$ suite of programs were used for the calculation and visualization of the topological properties of $\rho(\mathrm{r})$ and $\nabla^{2} \rho(\mathrm{r})$.

Conformational analysis of $\mathrm{Ni}^{\mathrm{II}}$ complexes with NTA and NTPA was done in solvent with the aid of Macromodel of the Schrödinger Maestro software package. ${ }^{45}$ Macromodel- $^{-}$ 
generated structures (OPLS 2005 force field was used), referred to as MM/MD structures, were fully energy-optimized in Gaussian in solvent to generate Gibbs free energy values used in the computation of formation constants and perform the topological analysis on those structures.

\section{Results and Discussion}

Computing a formation constant. The reaction employed here can be seen as a competition reaction $(\mathrm{CRn})$ between two ligands $\left(\mathrm{NTPA}=\mathrm{L}_{(1)}\right.$ and $\left.\mathrm{NTA}=\mathrm{L}_{(2)}\right)$ for nickel and it can be written as (charges are omitted for simplicity)

$\mathrm{NiL}_{(2)}\left(\mathrm{H}_{2} \mathrm{O}\right)_{2}+\mathrm{L}_{(1)}=\mathrm{NiL}_{(1)}\left(\mathrm{H}_{2} \mathrm{O}\right)_{2}+\mathrm{L}_{(2)} \quad \Delta G_{\mathrm{CRn}}(\mathrm{aq})$

where $\Delta G_{\mathrm{CRn}}(\mathrm{aq})$ is the change in the Gibbs free energy in solvent (water) for reaction 1. Complex formation reactions of nickel with NTPA, for which formation constant is to be computed, and NTA are shown, respectively, as eqs 2 and 3

$$
\begin{array}{ll}
\mathrm{Ni}\left(\mathrm{H}_{2} \mathrm{O}\right)_{6}+\mathrm{L}_{(1)}=\mathrm{NiL}_{(1)}\left(\mathrm{H}_{2} \mathrm{O}\right)_{2}+4 \mathrm{H}_{2} \mathrm{O} & \Delta G_{1}(\mathrm{aq}) \\
\mathrm{Ni}\left(\mathrm{H}_{2} \mathrm{O}\right)_{6}+\mathrm{L}_{(2)}=\mathrm{NiL}_{(2)}\left(\mathrm{H}_{2} \mathrm{O}\right)_{2}+4 \mathrm{H}_{2} \mathrm{O} & \Delta G_{2}(\mathrm{aq})
\end{array}
$$

where $\Delta G_{\mathrm{n}}(\mathrm{aq})$ is the change in the Gibbs free energy for the complex formation reactions 2 and 3 in solvent. The change in Gibbs energies for each complexation reaction (eqs 2 and 3) can be written, respectively, as

$$
\begin{aligned}
& \Delta G_{1}(\mathrm{aq})=G_{\mathrm{aq}}\left(\mathrm{NiL}_{(1)}\left(\mathrm{H}_{2} \mathrm{O}\right)_{2}\right)+4 G_{\mathrm{aq}}\left(\mathrm{H}_{2} \mathrm{O}\right)-G_{\mathrm{aq}}\left(\mathrm{Ni}\left(\mathrm{H}_{2} \mathrm{O}\right)_{6}\right)-G_{\mathrm{aq}}\left(\mathrm{L}_{(1)}\right) \\
& \Delta G_{2}(\mathrm{aq})=G_{\mathrm{aq}}\left(\mathrm{NiL}_{(2)}\left(\mathrm{H}_{2} \mathrm{O}\right)_{2}\right)+4 G_{\mathrm{aq}}\left(\mathrm{H}_{2} \mathrm{O}\right)-G_{\mathrm{aq}}\left(\mathrm{Ni}\left(\mathrm{H}_{2} \mathrm{O}\right)_{6}\right)-G_{\mathrm{aq}}\left(\mathrm{L}_{(2)}\right)
\end{aligned}
$$

The competition reaction of interest (eq 1) can be obtained from subtracting eq 3 from 2, and hence from subtracting eq 5 from 4 one obtains expressions for the change in Gibbs free energy applicable to this competition reaction

$$
\begin{aligned}
& \Delta G_{\mathrm{CRn}}(\mathrm{aq})=\Delta G_{1}(\mathrm{aq})-\Delta G_{2}(\mathrm{aq}) \\
& \Delta G_{\mathrm{CRn}}(\mathrm{aq})=G_{\mathrm{aq}}\left(\mathrm{NiL}_{(1)}\left(\mathrm{H}_{2} \mathrm{O}\right)_{2}\right)+G_{\mathrm{aq}}\left(\mathrm{L}_{(2)}\right)-G_{\mathrm{aq}}\left(\mathrm{L}_{(1)}\right)-G_{\mathrm{aq}}\left(\mathrm{NiL}_{(2)}\left(\mathrm{H}_{2} \mathrm{O}\right)_{2}\right.
\end{aligned}
$$

where the uncertainties related to $G_{\mathrm{aq}}\left(\mathrm{H}_{2} \mathrm{O}\right)$ and $G_{\mathrm{aq}}\left(\mathrm{Ni}\left(\mathrm{H}_{2} \mathrm{O}\right)_{6}\right)$ are no longer applicable as these terms cancel of. 
Eq 7 was used to calculate $\Delta G_{\mathrm{CRn}}(\mathrm{aq})$ of the competition reaction (eq 1) from appropriate Gibbs energies obtained for fully solvent-optimized structures of the ligands and complexes involved. The value for $\Delta G_{2}(\mathrm{aq})$ was obtained from well-known relationship

$$
\Delta G(\mathrm{aq})=-\mathrm{RT} \ln K
$$

using the reported formation constant ${ }^{2}$ (at $25^{\circ} \mathrm{C}, \mu=0.1 \mathrm{M}$ ) of the nickel complex with NTA. Once $\Delta G_{\mathrm{CRn}}(\mathrm{aq})$ and $\Delta G_{2}(\mathrm{aq})$ have been calculated, the value of $\Delta G_{1}(\mathrm{aq})$, which is needed to calculate the formation constant of the nickel ML complex with NTPA from eq 8, was obtained from eq 9

$$
\Delta G_{1}(\mathrm{aq})=\Delta G_{\mathrm{CRn}}(\mathrm{aq})+\Delta G_{2}(\mathrm{aq})
$$

From the computational protocol described above it follows that structures of four components seen in the competition reaction (eq 1) must be generated and optimized in solvent. We have shown recently that the best theoretically computed protonation constants are obtained when lowest energy conformers of molecules (ligands) are used. ${ }^{46,47}$ The lowest energy conformers of the free ligands NTPA and NTA reported by us recently ${ }^{46,47}$ were used in this work - Figure 1. It was of interest and utmost importance to find out whether, and to want extent, the selection of a structure from a pool of conformers of nickel complexes also plays a role in the prediction of formation constant. Our focus was on two parameters, the value of the Gibbs free energy of the energy-optimized complex, $G(\mathrm{aq})$ (a thermochemical parameter) and a combination of signs and values of dihedral angles Ni-O-C-C (a structural, or conformational, parameter related to the three acidic arms involved in the formation of chelating rings). Initially, we have constructed NiNTA and NiNTPA complexes (called further self-constructed complexes, S-c) and optimized them at the B3LYP/6-311+G(d,p) level of theory in solvent (PCM/UA0). These DFT-optimized structures were submitted to Schrödinger Maestro for complete conformational analysis in solvent. A large number of conformers was generated and they were grouped according to the combination of signs of dihedral angles Ni-O-C-C (following the number of O-atoms) in the two complexes. Several MM/MD-generated conformers, that differed significantly in the value of dihedral angles, were selected from each 
group and they were submitted for a complete solvent optimization in Gaussian using the B3LYP/6-311++G(d,p) level of theory and CPCM/UAKS solvation model.

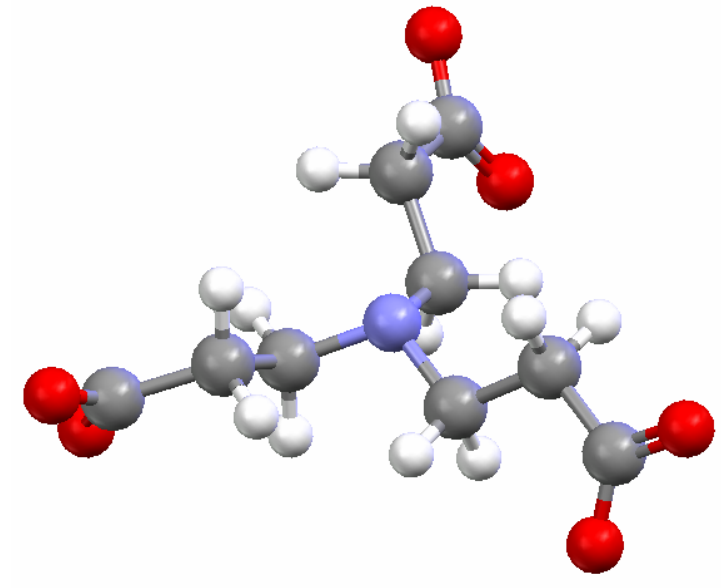

NTPA

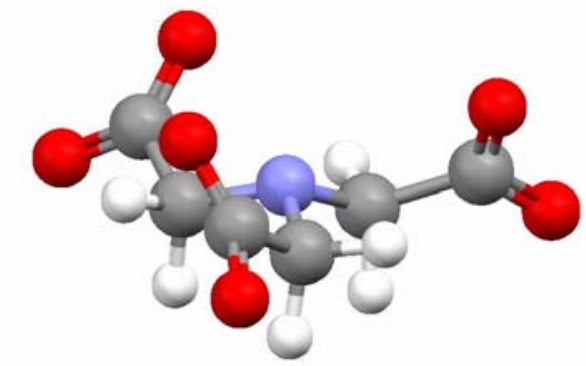

NTA

Figure 1. Lowest energy solvent-conformers ${ }^{46,47}$ of the free ligands used in computing of the NiNTPA formation constant.

In all cases, the values of dihedral angles of the MM/MD conformers decreased dramatically after optimization in Gaussian, sometimes by more than 40 degrees. Except few cases, the combination of signs of dihedral angles was preserved. From a number of Gaussian-optimized conformers, only few lowest-energy structures were selected for further studies. Full sets of NiNTA and NiNTPA conformers are shown in Figures S1 and S2 (Supplementary Information) and examples of nickel complexes, showing numbering of atoms, are shown in Figure 2.

Several tests were run in computing $\log K_{1}$ of the NiNTPA complex - selected results are presented in Table 1. In all tests the Gibbs free energies of the lowest energy ligand conformers, seen in Figure 1, were used. Initially, $G(\mathrm{aq})$ values obtained for the S-c conformer were used (Test 1 in Table 1) and, on average, $\log K_{1}=2.85 \pm 0.1$ was obtained (the reported ${ }^{2}$ experimental value is 5.8 at $30^{\circ} \mathrm{C}$ and $\mu=0.1 \mathrm{M}$ ). The error in $\log K_{1}$ of about $3 \log$ units is also observed in computed dissociation constants of singly protonated ligands from the DFTbased protocols. ${ }^{48-50}$ No improvement in computed $\log K_{1}$ value was obtained when CA1 conformers of NiNTA and NiNTPA were used - Test 2 in Table 1. Next, we have selected the lowest in DFT-energy conformers of complexes in which the signs of dihedral angles have not 
changed after optimization in Gaussian (B3LYP/6-311++G(d,p) using CPCM/UAKS) - see Test 3 .

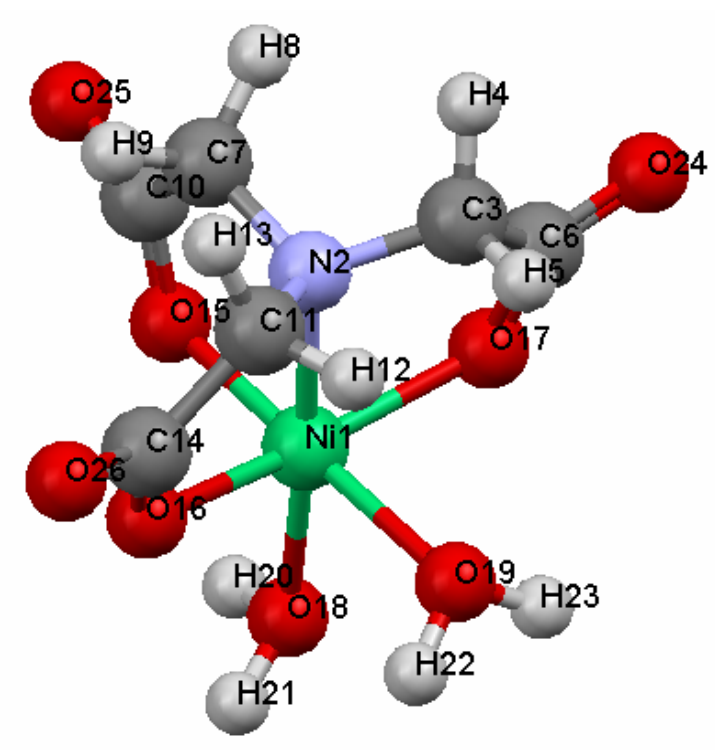

NiNTA, CA18

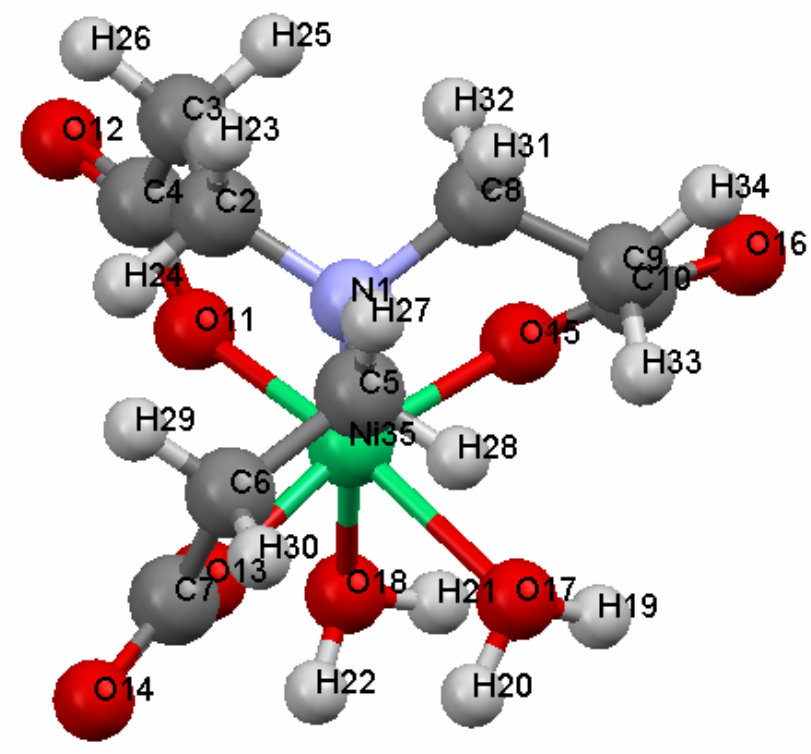

NiNTPA, S-c

Figure 2. Numbering of atoms in complexes discussed in this work. CA18 - initial MM/MD structure comes from the conformational MM/MD analysis. S-c is the self constructed complex.

An excellent theoretical value of $\log K_{1}=4.75$ was obtained. In the last test (Test 4 ) we have used the lowest energy conformers found after optimization in Gaussian, regardless whether signs of dihedral angles were preserved; CA18 of NiNTA and CA3 of NiNTPA (here the sign of dihedral angles changed after DFT optimization). The theoretically computed formation constant, $\log K_{1}=5.15$, can be seen as of analytical quality. From the analysis of results shown in Table 1 we came to the following conclusions: (i) the proposed protocol of computing formation constant works exceptionally well and most likely can be used for other metal complexes when two ligands of similar structures are involved; (ii) there is no need to involve expensive (time and hardware) high level theories; results obtained can be seen as excellent; (iii) it is of a paramount importance to use lowest energy conformers of ligands and metal complexes; (iv) a quick conformational search, involving MM/MD modeling, serves the purpose well, but carefully selected MM/MD-structures must be fully optimized by ab initio techniques as the MM/MD-generated energies as well as structures do not correlate well at all with those generated by Gaussian; (v) the combination of signs as well as values of dihedral 
angles (M-O-C-C) does not seem to have any significant influence on the computed formation constant.

In the above protocol a simple, but extensive conformational search was used. We wondered if it would be possible to develop somewhat simpler procedure. Two lowest in $G(\mathrm{aq})$ structures of NiNTA and NiNTPA found at the B3LYP/6-311++G(d,p) level of theory and CPCM/UAKS solvation model were used for further studies. The metal ion and two water molecules were removed from these structures and the remaining ligand, as present in a complex, was submitted for energy optimization in solvent at the same level of theory. We worked on the assumption that this should result in the 'energy-relaxed', possibly the lowest energy, ligand structure. Examples of the resultant conformers of the 'energy-relaxed' NTA and NTPA ligands are shown in Figure S3 and computed formation constants are shown in Table S1 (Supplementary Information). In general, good predictions of the formation constant were obtained and, when averaged, we obtained $\log K_{1}=3.9 \pm 0.5$ that differs from the experimental value by less than $2 \log$ units. This simplified approach resulted in a reasonable formation constant but clearly the most accurate results can only be obtained when lowest energy components are used.

Structural analysis. An excellent prediction in the formation constant of NiNTPA gave us an assurance that computationally generated structures of nickel complexes with NTA and NTPA can be used for further analysis in search of structural and physical properties controlling stability of complexes formed. Structural analysis (Table S2, Supplementary Information) involved several conformers, not only those that generated the best prediction in the formation constant. It is seen in Table S2 that regardless of the conformational structure, the coordination bond lengths follow the order $\mathrm{Ni}-\mathrm{O}^{-}<\mathrm{Ni}-\mathrm{N}<\mathrm{Ni}-\mathrm{OH}_{2}$. The Ni-N bond length (on average $2.094 \pm 0.007 \AA$ ) in the stronger NiNTA complex is shorter than in NiNTPA (on average $2.125 \pm 0.003 \AA$ ), but interestingly, on average shorter $\mathrm{Ni}^{-} \mathrm{O}^{-}$bonds are present in the NiNTPA conformers $(2.044 \AA)$. However, the middle chelating arm (with O15-atom) always forms the shortest $\mathrm{Ni}^{-} \mathrm{O}^{-}$bond $(2.031 \pm 0.003 \AA)$ and it is also shorter when compared with Ni$\mathrm{O}^{-}$bonds in the NiNTPA conformers. In case of the coordinated water molecules, they are closer to the central metal ion, on average by about $0.05 \AA$, in the NiNTA conformers and the axial water molecule (with O18-atom) placed opposite to the $\mathrm{N}$-atom is always closer to the central metal ion in both complexes, NiNTA and NiNTPA. 
Bite angles between the central metal ion and donor atoms in the ligands are, on average, $83^{\circ}$ in $5 \mathrm{~m}-\mathrm{CRs}$ and $94^{\circ}$ in $6 \mathrm{~m}-\mathrm{CRs}$ (the difference is only $11^{\circ}$ ). It has been suggested ${ }^{1}$ that the minimum-strain geometry for chelate ring of size five involving neutral oxygen and neutral nitrogen donors has a bite angles, O-M-O and N-M-N, of 58 and $69^{\circ}$, respectively, and for chelate ring size of six the ideal bite angles are 95 and $109.5^{\circ}$, respectively; the difference in the relevant ideal bite angles is about $40^{\circ}$. Even though carboxylic rather than neutral oxygen atoms are involved here, it was of interest to compare these theoretical bite values with computed ones. The computed ${ }^{-} \mathrm{O}-\mathrm{M}-\mathrm{N}$ bite angle $\left(83^{\circ}\right)$ in NiNTA is much larger (on average by about $20^{\circ}$ ) than the above ideal values for the $5 \mathrm{~m}$-CRs and the largest bite angle of $85.2^{\circ}$ is

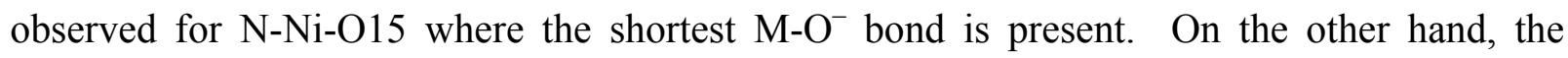
computed bite angle of $94^{\circ}$ in the $6 \mathrm{~m}-\mathrm{CRs}$ in NiNTPA is comparable with the minimum-strain geometry for this chelating ring. This analysis suggests that one should expect the ligand in the NiNTPA complex be less strained and hence this complex might possibly be stronger than NiNTA, but the experimental and computed $\log K_{1}$ values do not support that.

All torsions in NiNTA are small indicating that acetate arms are almost in plane, but a significant variation in torsion is observed in NiNTPA. In general, the dihedral angles (M-OC-C), their sign and value, do not seem to have an obvious and significant influence on computed formation constant. From analysis of data in Table S2 it would follow that (i) the structural analysis has not provided an evidence in support of the observed differences in stability of the NiNTA and NiNTPA complexes, and (ii) the structural properties of all conformers do not differ much hence one could use any NiNTA and NiNTPA conformer for comparative studies between the two complexes.

The QTAIM analysis. The quantum theory of atoms in molecules of Bader ${ }^{22}$ has been utilized to explore various interactions in a molecular system and its ability to identify these interactions between inter-atomic regions in terms of bond critical points and the formation of ring surfaces characterized by ring critical points is well documented. ${ }^{51-54}$ Examples of molecular graphs generated from the QTAIM analysis are show in Figure 3. As expected, three structural 5m-CR with the requisite RCPs are present but no additional intramolecular interactions were found in the NiNTA conformers. The results of the topological analysis (the values of the electron density, $\rho(\mathrm{r})$, and its Laplacian, $\nabla^{2} \rho(\mathrm{r})$, at the bond and ring critical points) of the coordination bonded interactions (between $\mathrm{Ni}$ (II) and donor atoms in the ligands) 
and structural chelating rings in the NiNTA and NiNTPA complexes are presented in Tables S3 and S4, respectively, in Supplementary Information.

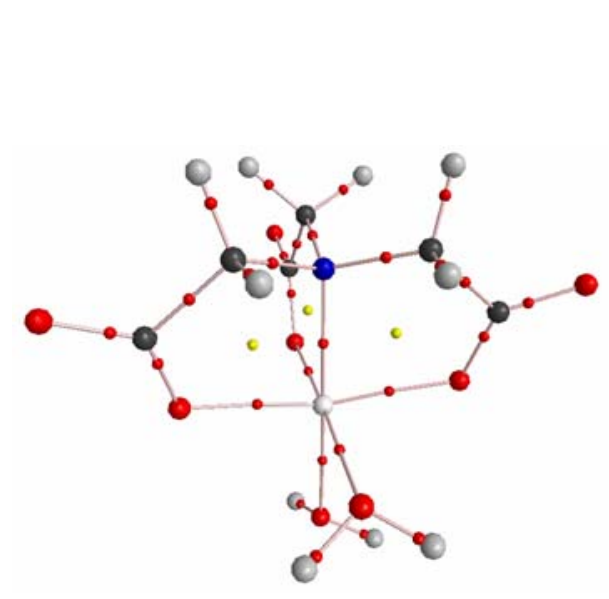

NiNTA, CA5

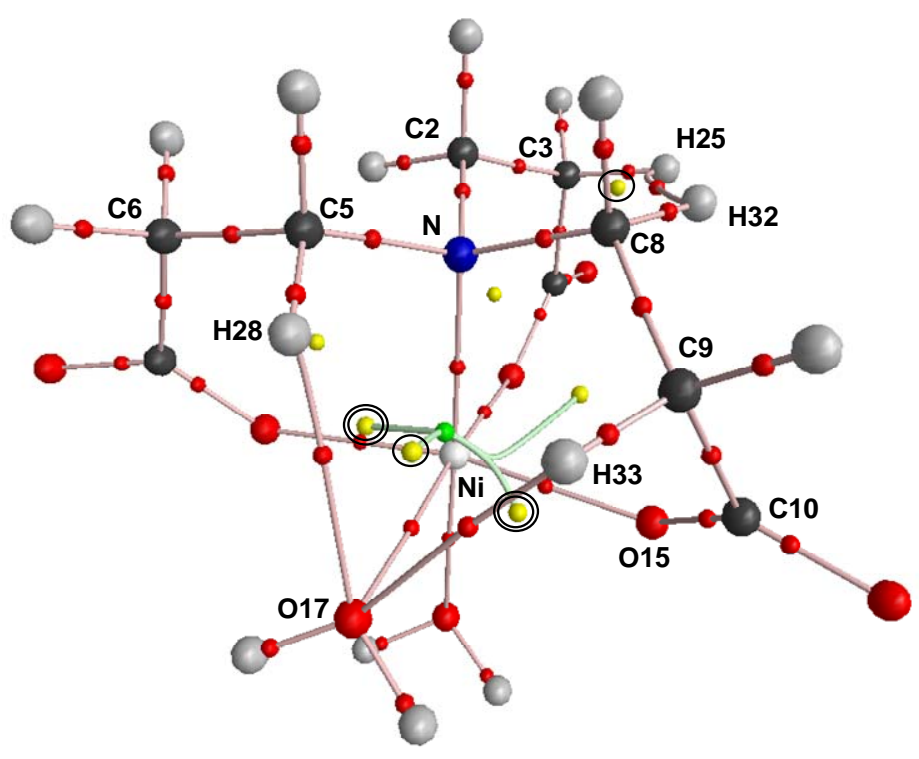

NiNTPA, S-c

Figure 3. Molecular graphs of the indicated conformers NiNTA and NiNTPA showing bond paths with BCPs (small spheres between two atoms), RCPs (three and seven in NiNTA and NiNTPA, respectively), and a cage critical point linked with four ring critical points in the NiNTPA complex. For more details see the text.

Values of $\rho_{\mathrm{BCP}}<0.10$ au are indicative of a closed-shell (i.e., predominantly non-covalent) interaction; ${ }^{55}$ it is usually accompanied by a relatively small and positive value of $\nabla^{2} \rho_{\text {BCP. }}{ }^{56}$ By contrast, for a shared (i.e., predominantly covalent) interaction, $\rho_{\text {BCP }}$ is usually $>0.1$ au $^{55}$ (or $>0.15 \mathrm{au}^{57}$ ) and $\nabla^{2} \rho_{\mathrm{BCP}}$, which may be positive or negative ${ }^{55}$ (but usually negative ${ }^{57}$ ), is typically of the same order as $\rho_{\mathrm{BCP}}$. As seen in Tables S3 and S4 in Supplementary Information, the values for the non-covalent framework are consistent with the closed-shell character of these bonds. The standard deviations in the average values of the electron density at BCPs, $\rho_{\mathrm{BCP}}$, and Laplacian of the electron density at BCPs, $\nabla^{2} \rho_{\mathrm{BCP}}$, are very small. It means that, as long as theoretically computed formation constant is reasonably close to the experimental value, all the conformers should be suitable for the structural and topological analyses.

The largest value of $\rho_{\mathrm{BCP}}$ was found for the Ni-N interactions, suggesting that they are the strongest, even though the $\mathrm{Ni}-\mathrm{N}$ bonds are significantly longer than the $\mathrm{Ni}-\mathrm{O}^{-}$bonds, e.g. by about $0.08 \AA$ in NiNTPA. Also, the Ni-N interaction is stronger in NiNTA as the electron 
density at the BCP (on average $\rho_{\mathrm{BCP}}=0.0728 \mathrm{au}$ ) is larger than in NiNTPA $\left(\rho_{\mathrm{BCP}}=0.0677 \mathrm{au}\right)$. The $\mathrm{M}-\mathrm{O}^{-}$bonds are almost of the same length in NiNTPA and the relevant distributions of the $\rho_{\mathrm{BCP}}$ and Laplacian values follow the same pattern. However, the values of $\rho_{\mathrm{BCP}}$ are slightly smaller (on average) in NiNTPA than in NiNTA which is opposite to the trend in this bond lengths. This suggests that the $\mathrm{Ni}^{-} \mathrm{O}^{-}$interactions are stronger in the NiNTA complex. Also, the coordinated water molecules add significantly more to the overall stability of NiNTA. Particularly in case of axial water molecule placed opposite to the $\mathrm{N}$-atom, the average value of $\rho_{\mathrm{BCP}}(0.0526 \mathrm{au})$ of the Ni-O18 bond path is significantly larger than in NiNTPA, $0.0458 \mathrm{au}$. The consistent trend in the strength of the $\mathrm{Ni}-\mathrm{N}$ and $\mathrm{Ni}^{-} \mathrm{O}^{-}$interactions when measured by $\rho_{\mathrm{BCP}}, \rho(\mathrm{r})_{\mathrm{Ni}(\mathrm{NTA})}>\rho(\mathrm{r})_{\mathrm{Ni}(\mathrm{NTPA})}$, agrees well with the strength of complexes formed, $\log K_{\mathrm{Ni}(\mathrm{NTA})}$ $>\log K_{\mathrm{Ni}(\mathrm{NTPA})}$ (a variation in the coordination bond length $(\mathrm{BL})$ vs. $\rho(\mathrm{r})$ values at different bond critical points is discussed in details in Supplementary Information, Figure S4).

Large differences are observed in topological properties of the structural chelating rings in NiNTA and NiNTPA. The electron density at the RCPs of the 5m-CRs in NiNTA (on average, $\rho_{\mathrm{RCP}}=0.0227 \mathrm{au}$, Table S3, Supplementary Information) is almost twice as large as at the RCPs of the 6m-CRs in NiNTPA, Table S4 in Supplementary Information. Similar pattern is observed for the Laplacian values at RCPs in the two complexes. It appears that the chelating effect, when measured by the electron density at RCPs, is much stronger in NiNTA then in NiNTPA. The properties of RCPs were used to measure the strength of intramolecular H-bonding ${ }^{58}$ and, when in combination with other topological properties, as a predictive parameter of basicity of pyridines. ${ }^{59}$ It would be of great interest and importance to study a large number of metal complexes with different ligands in order to find out if it would be possible to develop a new and physical measure of the chelating effect using $\rho_{\mathrm{RCP}}$ and $\nabla^{2}{ }_{\mathrm{RCP}}$ values. It is important to notice that, regardless how twisted the chelating arms are the values of $\rho(\mathrm{r})$ and Laplacian at the RCPs are almost the same in all relevant conformers after optimization in Gaussian. As an example, the average $\rho_{\text {RCP }}$ value of all chelating arms in four conformers of NiNTA is $0.0227 \pm 0.0004$ au. Even smaller standard deviation in the average $\rho_{\text {RCP }}$ is observed for the NiNTPA conformers. This is in a very strong support of the above supposition that to understand physical and geometrical parameters controlling complex stability, one does not have to generate structures that generate formation constant of analytical quality. 
Structural and topological properties of additional, non-structural, intramolecular interactions and close contacts 'ignored' by the QTAIM analysis in the NiNTPA conformers are of interest now - see Table 2. There are consistent features present in each of the five conformers of NiNTPA and the self-constructed complex seen in Figure 3 will be used for illustration purposes: (i) there are three intramolecular close contacts $(25 \mathrm{H} \cdots \mathrm{H} 32,17 \mathrm{O} \cdots \mathrm{H} 28$, and $17 \mathrm{O} \cdots \mathrm{H} 33$ ) which have QTAIM-defined bond paths and BCPs; (ii) in addition to three structural $6 \mathrm{~m}-\mathrm{CRs}$, four non-structural rings are formed with RCPs placed well inside the rings - these RCPs are marked with circles; (iii) two non-structural rings incorporate the coordinated Ni-atom hence they can be seen as chelating rings and their RCPs are marked with double circles (NiNTPA has 5 chelating rings in total, regardless of a conformer analyzed); (iv) one of the non-structural chelating rings is always a 5m-ring (N-C5-H28-O17-Ni) and the electron density at its RCP is about 75-80\% of what is observed in the structural 5m-CRs; (v) the 6mnonCR is always generated by the presence of the $\mathrm{CH} \cdots \mathrm{HC}$ short contact (classically, a $\mathrm{H}$ clash) and this ring has the largest electron density at the RCP among all the rings; (vi) only one cage is formed, which is characterized by a cage critical point, CCP - see Figure 3 where paths connecting the four RCPs with the CCP are shown; (vii) there are two short intramolecular contacts that were 'ignored' by the QTAIM analysis; a bond path is not present.

In each conformer there are two $\mathrm{CH} \cdots \mathrm{HC}$ contacts and one $\mathrm{CH} \cdots \mathrm{O}$ contact, except the S-c conformer which has two $\mathrm{CH} \cdots \mathrm{O}$ and one $\mathrm{CH} \cdots \mathrm{HC}$ contact. Because QTAIM interpreted the $\mathrm{H}$-clashes and $\mathrm{CH} \cdots \mathrm{O}$ contacts as bonded interactions ${ }^{22,51,60-65}$ (bond paths are present), they resulted in four additional non-structural rings with the requisite RCPs. It is well-established that all the additional intramolecular interactions with BCPs, RCPs, as well as CCP contribute locally to the overall stability of a molecule because a bond path in QTAIM indicates the presence of a preferred quantum-mechanical exchange channel and the inter-atomic exchangecorrelation energy always stabilizes the local interaction. ${ }^{54}$ The stabilization may not be negligible; it has been demonstrated that for $\rho_{\mathrm{BCP}}$ values in the range of 0.01 au (as is the case here - Table 2) the formation of a $\mathrm{H}-\mathrm{H}$ bond has a stabilizing effect ${ }^{51,61}$ of up to $10 \mathrm{kcal} \mathrm{mol}^{-1}$ and this pairwise additive stabilization does not depend on the attractive or repulsive nature of the classical interaction between the atoms' charge densities. These contributions in NiNTPA appear to be significant when electron densities at the BCPs, RCPs, and CCPs are analyzed in Table 2. For instance, the electron density at BCPs of the $\mathrm{H} \cdots \mathrm{H}$ and $\mathrm{CH} \cdots \mathrm{OH}_{2}$ interactions 
(between 0.0088 and $0.0144 \mathrm{au}$ ) are comparable with the $\rho_{\mathrm{RCP}}$ values of the structural $6 \mathrm{~m}-\mathrm{CRs}$ (on average $0.0122 \mathrm{au}$ ). Also, each conformer forms a non-structural $6 \mathrm{~m}$-ring with $\rho_{\text {RCP }}$ of about $0.0134 \mathrm{au}$, which is larger than $\rho_{\mathrm{RCP}}$ in the structural $6 \mathrm{~m}-\mathrm{CRs}$. This suggests that the energy stabilizing effect of this non-structural ring is the strongest among all, structural and non-structural, rings in this complex. Interestingly, this ring is formed due to the presence of the $\mathrm{H} \cdots \mathrm{H}$ interactions and the electron density at their BCPs follows the expected linear relationship between $\rho_{\mathrm{BCP}}$ and a bond length - see empty circles in Figure S5, Supplementary Information.

To verify whether $\mathrm{H} \cdots \mathrm{H}$ contacts specified above are attractive, the physical properties of relevant $\mathrm{H}$-atoms were analyzed in the CA4 and CA3 conformers of NiNTPA. These H-atoms form $\mathrm{a}-\mathrm{CH}_{2}-$ fragment of the chelating arms of the ligand NTPA. The atomic energy of $\mathrm{H}-$ atoms involved in the $\mathrm{H} \cdots \mathrm{H}$ interaction is usually lower when compared with the energy of a geminal H-atom, $\Delta E(\mathrm{H})<0$ in Table 3. However, the local stabilizing energy contribution (e.g. in the CA4 conformer, $(\mathbf{H 2 9}-\mathrm{H} 30)+(\mathbf{H 2 6}-\mathrm{H} 25)=E_{\text {stab }}=-7.75 \mathrm{kcal} \mathrm{mol}^{-1}$; atoms involved in the intramolecular interactions are in bold) is always negative and can be interpreted as an attractive interaction, hence should be seen as the $\mathrm{H}-\mathrm{H}$ bond. ${ }^{51}$ There are two $\mathrm{H}-\mathrm{H}$ bonds in each conformer and their local stabilizing contribution, $E_{\text {stab }}$, is about $7 \mathrm{kcal}$ $\mathrm{mol}^{-1}$. This is equivalent to about four $\log K_{\mathrm{ML}}$ units $\left(1.36 \mathrm{kcal} \mathrm{mol}^{-1}\right.$ is equivalent to $1 \log K$ unit). The presence of $\mathrm{H}-\mathrm{H}$ bonds can also be confirmed by the analysis of the atomic energies, $E(\mathrm{H})$, of the same $\mathrm{H}$-atoms when they are and are not involved in the intramolecular interaction $^{51}$ - Table 4. As an example, the energy of H26- and H25-atoms is lowered $(\Delta E)$ by 4.78 and $4.35 \mathrm{kcal} \mathrm{mol}^{-1}$, respectively, when they are involved in the $\mathrm{H}-\mathrm{H}$ bonding in the CA4 and CA3 conformers, respectively. This kind of intramolecular interaction, when identified in metal complexes, has been interpreted as a destabilizing non-bonded steric repulsion responsible for the decrease in stability of a complex. This does not seem to hold because according to QTAIM, the $\mathrm{CH} \cdots \mathrm{HC}$ close contacts, or $\mathrm{H}-\mathrm{H}$ bonds, contribute to the overall stability of NiNTPA as much as the formation of the structural 6m-CRs in the NiNTPA complex.

A similar analysis was performed on the $\mathrm{CH} \cdots \mathrm{O}$ close contacts present in all conformers of the NiNTPA complex - Table 5. It is seen that the atomic energy of H28- and H31-atoms, which are involved in the $\mathrm{CH} \cdots \mathrm{O}$ intramolecular interactions in the $\mathrm{CA} 4$ and $\mathrm{CA} 3$ conformers, 
respectively, are lower in energy by 3.33 and $5.13 \mathrm{kcal} \mathrm{mol}^{-1}$ when compared with the relevant geminal H27- and H32-atoms. Also the atomic energy of theH28- and H31-atoms is lowered by 2.56 and $4.25 \mathrm{kcal} \mathrm{mol}^{-1}$ when they are involved the $\mathrm{CH} \cdots \mathrm{O}$ interaction in the $\mathrm{CA} 4$ and CA3 conformers, respectively (Table 6), Results obtained strongly indicate that this interaction is attractive and should be viewed as an additional intramolecular $\mathrm{CH}-\mathrm{O}$ bond. ${ }^{63-65}$

Each conformer of the NiNTPA complex has two close contacts, either $\mathrm{CH} \cdots \mathrm{HC}$ or $\mathrm{CH} \cdots \mathrm{O}$, which were not seen by the QTAIM analysis as additional bonds even though the necessary condition, the distance criterion, is satisfied. For instance, the distance between two H-atoms, $\mathrm{CH} 24 \cdots \mathrm{H} 29 \mathrm{C}$, in S-c conformer is $2.108 \AA$ and this is significantly shorter than similar contact

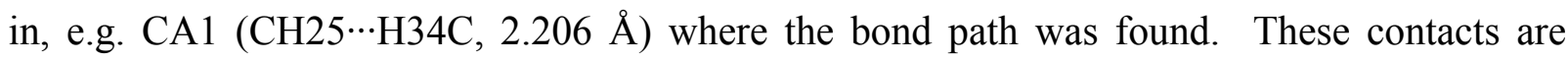
marked as solid squares in Figure S5 to indicate the expected $\rho_{\mathrm{BCP}}$ values if the bond paths were present, but clearly the electron density between the two relevant atoms must be significantly lower. The reason for that is not quite obvious at present, although it has been demonstrated recently that an inter-atomic distance smaller than the sum of the van der Waals radii is not a sufficient condition for the bond path formation. This is because the directional exchange interaction may dominate over non-directional classical electrostatic interactions. ${ }^{54}$ In such a case the distance rule is invalid and examples of atoms that bond preferentially to others that are further, rather than to closer neighbors, are known. ${ }^{66,67}$ It has been suggested recently that the presence of the five- and six-membered reinforcing rings alters the intramolecular interactions in a molecule; ${ }^{68}$ the QTAIM analysis has not predicted the formation of $\mathrm{H}-\mathrm{H}$ bonds in $\mathrm{Cyp}_{2}-\mathrm{EN}$ ( $N, N$ '-bis(2-hydroxycyclopentyl)-ethane-1,2-diamine) even though the distance criterion was met. This was attributed to the $\rho_{\mathrm{RCP}}$ and $\nabla^{2} \rho_{\mathrm{RCP}}$ values of the cyclopentyl moiety in $\mathrm{Cyp}_{2}-\mathrm{EN}$ that were twice as large as those of the cyclohexyl moiety in $\mathrm{Cy}_{2}$-EN ( $N, N^{\prime}$-bis(2-hydroxycyclohexyl)-ethane-1,2-diamine). A postulate was made that the increased electron density within the 5-membered reinforcing ring is responsible for the absence of the preferential quantum-mechanical exchange channels between atoms that meet the distance criterion.

It was of utmost interest and importance to find physical properties responsible for the absence of bond paths in NiNTPA where the distance criterion was met. As an example, let us analyze the S-c conformer of NiNTPA (Figure 4). The CH24*H29C contact $(2.108 \AA$, marked by the dotted line) involves C2- and C6-atoms in the C2-C3-C4-O11 and C5-C6-C7- 
O13 arms, respectively. The C2-atom (to which H24-atom is bonded) is involved in the structural chelating N-C2-C3-C4-O11-Ni ring with the RCP marked with double circle and non-structural and non-chelating N-C2-C3-H25-H32-C8 ring with the RCP marked with a single circle in Figure 4. The C6-atom (to which H29-atom is bonded) is involved only in the structural N-C5-C6-C7-O13-Ni chelating ring with the RCP marked with double circle. However, the C5-atom, which is the member of this structural ring, is involved in two nonstructural rings, namely the chelating $\mathrm{N}-\mathrm{C} 5-\mathrm{H} 28-\mathrm{O} 17-\mathrm{Ni}$ and non-chelating $\mathrm{N}-\mathrm{C} 5-\mathrm{H} 28-\mathrm{O} 17-$ H33-C9-C8 ring with RCP marked with a single circle in Figure 4.

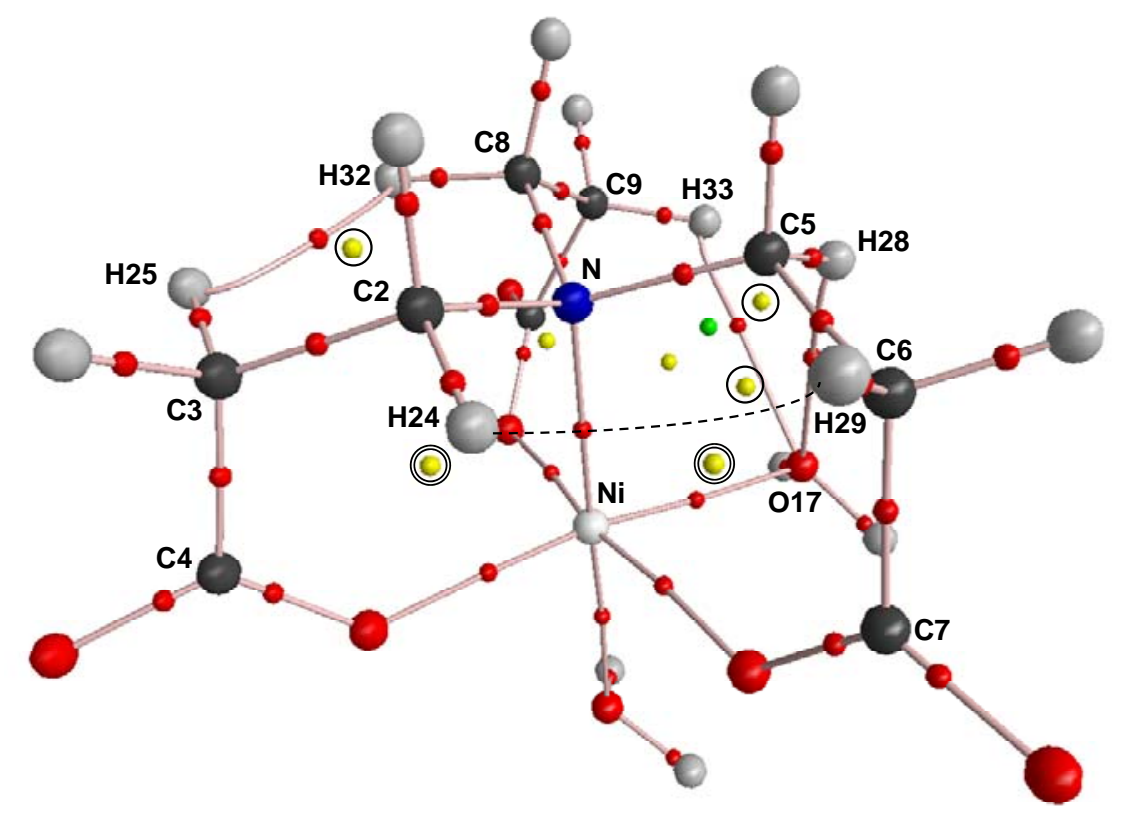

Figure 4. Molecular graph of the S-c NiNTPA complex showing $\mathrm{CH} \cdots \mathrm{HC}$ close contact (dashed line) which does not have a bond path. The ring critical points of the structural and nonstructural rings are marked with double-line and single-line circles, respectively.

It is seen in Table 2 that the $\rho_{\mathrm{RCP}}$ and $\nabla^{2} \rho_{\mathrm{RCP}}$ values of the non-structural ring N-C2-C3-H25H32-C8 (0.0134 and 0.0621 au, respectively) are larger when compared with the structural chelating N-C2-C3-C3-O11-Ni ring (0.0120 and 0.0548 au, respectively). It appears that the formation of an adjacent ring with a larger electron density at the RCP, when compared with the ring to which the atoms forming a close contact belong, resulted in the absence of the bond path. This observation correlates well with findings reported recently ${ }^{68}$ and when combined with the formation of two additional non-structural rings involving the part of the arm in which C6-atom is present, can be used to explain the absence of the preferential quantum-mechanical 
exchange channels between H24- and H29-atoms that meet the distance criterion. Similar observation applies to the $\mathrm{CH}^{\cdots} \mathrm{OH}_{2}$ contacts as it is explained in details in Supplementary Information, Figure S6. As far as we know, this is the first attempt to explain the absence of BCPs in close atomic intramolecular contacts in metal complexes using the $\rho_{\mathrm{RCP}}$ and $\nabla^{2} \rho_{\mathrm{RCP}}$ values.

Strain energy analysis. The additional three intramolecular bonded interactions (the $\mathrm{H}-\mathrm{H}$ and $\mathrm{CH}-\mathrm{O}$ bonds), four rings and one cage were found by the QTAIM analysis only in NiNTPA. Then why the NiNTPA complex is so much weaker? In search for the answer, the energies of the as-in-complex NTPA in the NiNTPA conformers were computed using a single point frequency calculation (SPFC). The zero-point vibrational energy corrected electronic energy of as-in-complex ligand, $E_{\mathrm{ZPVE}}\left(\mathrm{L}_{\text {comp }}\right)$, was compared with the electronic energy of the relevant lowest-energy conformer of the free ligands, $E_{\mathrm{ZPVE}}\left(\mathrm{L}_{\text {free }}\right)$ seen in Figure 1. All the calculations were performed at the same level of theory in solvent and results are shown in Table 7. It is seen that both ligands (NTA and NTPA) are strained in complexes, $E_{\mathrm{s}}=E_{\mathrm{ZPVE}}\left(\mathrm{L}_{\mathrm{comp}}\right)$ $E_{\text {ZPVE }}\left(\mathrm{L}_{\text {free }}\right)$. At the same time, NTPA is significantly more strained than NTA, by about 16 $\mathrm{kcal} \mathrm{mol}^{-1}$, which corresponds to a decrease in the formation constant by about 12-13 log units. One can argue that the absolute strain energy values, even though informative, should not be used directly for a rigorous evaluation because the ligands contain significantly different number of atoms. However, the ratio of $E_{\mathrm{s}}(\mathrm{NTPA}) / E_{\mathrm{s}}(\mathrm{NTA})$ might have some physical significance - Table 7. An arbitrary selection was used by pairing S-c, CA1, second lowest and the lowest energy conformers of the NiNTA and NiNTPA complexes. There are many possible combinations, but all of them would give very much the same overall result. It was interesting to see that the average $E_{\mathrm{s}}$ ratio of 1.9 (obtained at the RB3LYP/6-311++G(d,p) level of theory using the CPCM-UAKS solvation model) correlates very well with the ratio of the $\log K_{1}$ values (NTA/NTPA) from the experiment, 1.98. It suggests that the larger strain energy in NTPA is the main reason for the decrease in the stability of the NiNTPA complex.

We have established that most of the intramolecular interactions (as discussed above in the QTAIM analysis) are also present in the ligand as a fragment of a complex - see Figure S7, Table S5 and details in Supplementary Information. The degree to which a ligand is strained must depend on structural configuration it has to adopt to form a complex. In this case, the geometry around Ni-atom has not changed significantly in NiNTA and NiNTPA suggesting 
that the preferred by Ni(II) geometry (within some limits) imposed structural configuration on the ligand NTPA. One might also assume that the local bonded interactions significantly decreased the strain energy in the as-in-complex ligand; NiNTPA most likely would be much weaker, or even did not form at all, if the $\mathrm{H}-\mathrm{H}$ and $\mathrm{CH}-\mathrm{O}$ bonds were not formed; the same close contacts are also observed in crystallographic structure of the NiNTPA complex. ${ }^{69}$

\section{Conclusions.}

It has been shown that it is possible to theoretically compute the $\log K_{1}$ value of analytical quality from the competition reaction between two ligands (NTA and NTPA) for a metal ion $\mathrm{Ni}(\mathrm{II})$ using the $G(\mathrm{aq})$ values of the lowest energy conformers of the ligands and complexes and experimental $\log K_{1}$ value of NiNTA. The described mathematical protocol is of general nature and should allow computing formation constants for many metal-ligand systems. A methodology, that does not involve a conformational analysis of ligands, has also been tested; high quality predictions in $\log K_{1}$ values were obtained and they differed from the experimental data by less than 2 log units. It appears that QTAIM theory is a powerful tool in understanding physical properties of complexes formed in solvent, such as nature of structural and non-structural intramolecular interactions; they were used to explain the differences in formation constants. It has been discovered that two kinds of intramolecular bonds, $\mathrm{H}-\mathrm{H}$ and $\mathrm{CH}-\mathrm{O}$, are formed in the NiNTPA complex; they contribute to the overall stability of the complex.. QTAIM proved that structural $\mathrm{H}$-clashes in NiNTPA are in fact the $\mathrm{H}-\mathrm{H}$ bonds and do not constitute a steric hindrance destabilizing NiNTPA. From results reported here it follows that the strength of bonded interactions should be estimated from $\rho_{\mathrm{BCP}}$ rather than the bond lengths. In both complexes $\mathrm{Ni}-\mathrm{N}>\mathrm{Ni}-\mathrm{O}^{-}$, but $\rho_{\mathrm{Ni}-\mathrm{N}}>\rho_{\mathrm{Ni}-\mathrm{O}}-$ even though the $\mathrm{Ni}-\mathrm{N}$ bond in NiNTPA is much longer (by about $0.08 \AA$ ) than the Ni-O $\mathrm{O}^{-}$bonds. An analysis of the strain energy in the as-in-complex NTPA ligand strongly suggests that much larger energy penalty is paid by NTPA than NTA; the strain energy ratio (NTPA/NTA) of 1.9 compares very well with the experimental ratio, 1.98, of $\log K_{1}$ values (NTA/NTPA). The computed strain energy in the NTPA ligand is significantly decreased by the presence of intramolecular bonds found from the QTAIM analysis meaning that in their absence NiNTPA would be much weaker or not formed at all. The stronger chelating power of the NTA ligand was tentatively ascribed to the strength of the structural chelating rings when measured by the $\rho(\mathrm{r})$ and $\nabla^{2} \rho(\mathrm{r})$ at the RCPs; 
these values were twice as large in structural 5m-CRs of NiNTA than in structural 6m-CRs of NiNTPA complexes. It is postulated that the absence of a bond path between atoms meeting the distance criterion is due to the formation of adjacent intramolecular rings that have larger electron density at RCPs when compared with the rings containing these atoms.

\section{Acknowledgments:}

Financial support of the National Research Foundation of South Africa and the University of Pretoria is highly appreciated.

Supporting Information Available: Full sets of NiNTA and NiNTPA conformers used to compute $\log K_{1}$ of NiNTPA; A simplified protocol used to compute a formation constant; Structural properties of the selected conformers of the NiNTA and NiNTPA complexes; The $\rho(\mathrm{r})$ and $\nabla^{2} \rho(\mathrm{r})$ values at BCPs and RCPs found by the QTAIM analysis for the bond paths of the coordination bonds and structural 5m-CRs in the conformers of the NiNTA and NiNTPA complexes; Relationship between the electron density at BCPs and coordination bond lengths; Relationship between the electron density at BCPs and intramolecular $\mathrm{H}-\mathrm{H}$ bond lengths; an analysis of the absence of the $\mathrm{CH}-\mathrm{O}$ bond path; Intramolecular interactions in NTPA as a fragment of the NiNTPA complex. This material is available free of charge via the Internet at http://pubs.acs.org 


\section{References}

(1) Martell, A. E.; Hancock, R. D. in Metal Complexes in Aqueous Solutions, Plenum Press, New York, 1996.

(2) NIST Standard Reference Database 46. NIST Critically Selected Stability Constants of Metal Complexes Database, Version 8.0, Data collected and selected by R. M. Smith and A. E. Martell, US Department of Commerce, National Institute of Standards and Technology, 2004.

(3) The IUPAC Stability Constants Database, http://www.iupac.org distributed and maintained by Academic Software, Sourby Old Farm, Timble, Otley, Yorks, LS21, 2PW, UK, (http://www.acadsoft.co.uk/scdbase/)

(4) Liptak, M. D.; Shields, G. C. Int. J. Quantum Chem. 2001, 85, 727-741.

(5) Liptak, M. D.; Gross, K. C.; Seybold, P. G.; Feldgus, S.; Shields, G. C. J. Am. Chem. Soc. 2002, 124, 6421-6427.

(6) Namazian, M.; Zakery, M.; Noorbala, M. R.; Coote, M. L. Chem. Phys. Lett. 2008, 451, $163-168$.

(7) Sang-Aroon, W.; Ruangpornvisuti, V. Int. J. Quantum Chem. 2008, 108, 1181-1188.

(8) da Silva, C. O.; da Silva, E. C.; Nascimento, M. A. C. J. Phys. Chem. A 1999, 103, 11194-11199.

(9) Kelly, C. P.; Cramer, C. J.; Truhlar, D. G. J. Phys. Chem. A 2006, 110, 2493-2499.

(10) Pliego Jr., J. R.; Riveros, J. M. J. Phys. Chem. A 2002, 106, 7434-7439.

(11) Benson, M. T.; Moser, M. L.; Peterman, D. R.; Dinescu, A. J. Mol. Struct. (THEOCHEM) 2008, 867, 71-77.

(12) Hancock, R. D.; Bartolotti, L. J. Chem. Soc. Chem. Commun. 2004, 534-535.

(13) Hancock, R. D.; Bartolotti, L. J. Inorg. Chem. 2005, 44, 7175-7183.

(14) Salehzadeh, S.; Bayat, M.; Ward, M. D. J. Phys. Chem. A 2008, 112, 4090-4094.

(15) Salehzadeh, S.; Shooshtari, A.; Bayat, M. Dalton Trans., 2009, 2865-2870.

(16) Hancock, R. D. Progr. Inorg. Chem. 1989, 36, 187-291.

(17) Hancock, R. D. Acc. Chem. Res. 1990, 23, 253-257.

(18) Shannon, R. D. Acta Crystallogr., Sect. A 1976, A32, 751-767.

(19) Hancock, R. D.; de Sousa, A. S.; Walton, G. B.; Reibenspies, J. H. Inorg. Chem. 2007, $46,4749-4757$. 
(20) Thom, V. J.; Fox, C. C.; Boeyens, J.C.A.; Hancock, R. D. J. Am. Chem. Soc. 1984, 106, 5947-5955.

(21) Hambley, T. W. J. Chem. Soc. Dalton Trans. 1986, 565-569.

(22) Bader, R. F. W. in Atoms in Molecules: A Quantum Theory, Clarendon Press, Oxford, 1990.

(23) Frisch, M. J.; Trucks, G. W.; Schlegel, H. B.; Scuseria, G. E.; Robb, M. A.; Cheeseman, J. R.; Montgomery, J. A.; Jr.; Vreven, T.; Kudin, K. N.; Burant, J. C.; Millam, J. M.; Iyengar, S. S.; Tomasi, J.; Barone, V.; Mennucci, B.; Cossi, M.; Scalmani, G.; Rega, N.; Pettersson, G. A.; Nakatsuji, H.; Hada, M.; Ehara, M.; Toyota, K.; Fukuda, R.; Hasegawa, J.; Ishida, M.; Nakajima, T.; Honda, Y.; Kitao, O.; Nakai, H.; Klene, M.; Li, X.; Knox, J. E.; Hratchian, H. P.; Cross, J. B.; Bakken, V.; Adamo, C.; Jaramillo, J.; Gomperts, R.; Stratmann, R. E.; Yazyev, O.; Austin, A. J.; Cammi, R.; Pomelli, C.; Ochterski, J. W.; Ayala, P. Y.; Morokuma, K.; Voth, G. A.; Salvador, P.; Dannenberg, J. J.; Zakrzewski, V. G.; Dapprich, S.; Daniels, A. D.; Strain, M. C.; Farkas, O.; Malick, D. K.; Rabuck, A. D.; Raghavachari, K.; Foresman, J. B.; Ortiz, J. V.; Cui, Q.; Baboul, A. G.; Clifford, S.; Cioslowski, J.; Stefanov, B. B.; Liu, G.; Liashenko, A.; Piskorz, P.; Komaromi, I.; Martin, R. L.; Fox, D. J.; Keith, T.; Al-Laham, M. A.; Peng, C. Y.; Nanayakkara, A.; Challacombe, M.; Gill, P. M. W.; Johnson, B.; Chen, W.; Wong, M. W.; Gonzalez, C.; Pople, J. A. Gaussian 03, Revision D.01; Gaussian, Inc., Wallingford, CT, 2004.

(24) GaussView 4.1.2, Gaussian, Inc.; Wallingford, CT, 2004.

(25) Saracino, G. A. A.; Improta, R.; Barone, V. Chem. Phys. Lett. 2003, 373, 411-415.

(26) Varadwaj, P. R.; Cukrowski, I.; Marques, H. M. J. Phys. Chem. A 2008, 112, 1065710666.

(27) Onsager, L. J. Am. Chem. Soc. 1936, 58, 1486-1493.

(28) Tapia, O.; Goscinski, O. Mol. Phys. 1975, 29, 1653-1661.

(29) Miertus, S.; Scrocco, E.; Tomasi, J. Chem. Phys. 1981, 55, 117-129.

(30) Miertus, S.; Tomasi, J. Chem. Phys. 1982, 65, 239-245.

(31) Tomasi, J.; Persico, M. Chem. Rev. 1994, 94, 2027-2094.

(32) Cramer, C. J.; Truhlar, D. G. Chem. Rev. 1999, 99, 2161-2200.

(33) Chipman, D. M. J. Phys. Chem. A. 2002, 106, 7413-7422.

(34) Zhan, C.-G.; Bentley, J.; Chipman, D. M. J. Chem. Phys. 1998, 108, 177-192.

(35) Klamt, A.; Schüürmann, G. J. Chem. Soc., Perkin Trans. 1993, 2, 799-805. 
(36) Andzelm, J.; Kölmel, C.; Klamt, A. J. Chem. Phys. 1995, 103, 9312-9320.

(37) Barone, V; Cossi, M. J. Phys. Chem. A 1998, 102, 1995-2001.

(38) Cossi, M.; Barone, V. J. Chem. Phys. 1998, 109, 6246-6254.

(39) Cossi, M.; Rega, N.; Scalmani, G.; Barone, V. J. Comput. Chem. 2003, 24, 669-681.

(40) McLean, A. D.; Chandler, G. S. J. Chem. Phys. 1980, 72, 5639-5648.

(41) Krishnan, R.; Binkley, J. S.; Seeger, R.; Pople, J. A. J. Chem. Phys. 1980, 72, 650-654.

(42) Keith, T. A. AIMAll, 08.05.04, http://aim.tkgristmill.com, 2008.

(43) Biegler-König, F.; Schönbohm, J.; Bayles, D. AIM2000, http:/www.gauss.fhbielefeld.de/aim 2000

(44) Biegler-König, F.; Schönbohm, J.; Bayles, D. J. Comp. Chem. 2001, 22, 545-559.

(45) Schrodinger, L. L. C. Schrodinger Maestro, 32nd Floor, Tower 45, 120 West Forty-Fifth Street, New York, 10036, 2003.

(46) Govender, K. K.; Cukrowski, I. J. Phys. Chem. A, 2009, 113, 3639-3647.

(47) Govender, K. K.; Cukrowski, I. J. Phys. Chem. A, 2010, 114, 1868-1878.

(48) Lim, C.; Bashford, D.; Karplus, M. J. Phys. Chem. 1991, 95, 5610-5620.

(49) Namazian, M.; Halvani, S.; Noorbala, M. R. J. Mol. Struct. (Theochem) 2004, 711, 1318.

(50) Cramer, C. J. in Essentials of Computational Chemistry; John Wiley \& Sons Ltd.: New York, 2002, pp. 371-372.

(51) Matta, C. F.; Hernandez-Trujillo, J.; Tang, T.-H.; Bader, R. F. W. Chem. Eur. J. 2003, 9, $1940-1951$.

(52) Grabowski, S. J.; Pfitzner, A.; Zabel, M.; Dubis, A. T.; Palusiak, M. J. Phys. Chem. B 2004, 108, 1831-1837.

(53) Matta, C. F.; Castillo, N.; Boyd, R. J. J. Phys. Chem. A 2005, 109, 3669-3681.

(54) Pendás, A. M.; Francisco, E.; Blanco, M. A.; Gatti, C. Chem. Eur. J. 2007, 13, 93629371.

(55) Bader, R. F. W.; Essén, H. J. Chem. Phys. 1984, 80, 1943-1960.

(56) Bone, R. G. A.; Bader, R. F. W. J. Phys. Chem. 1996, 100, 10892-10911.

(57) Bobrov, M. F.; Popova, G. V.; Tsirelson, V. G. Russ. J. Phys. Chem. 2006, 80, 584-590.

(58) Grabowski, S. J. Monatsh. Chem. 2002, 133, 1373-1380.

(59) Hawe, G. I.; Alkorta, I.; Popelier, P. L. A. J. Chem. Inf. Model. 2010, 50, 87-96. 
(60) Matta, C. F. Hydrogen-Hydrogen Bonding: The Non-Electrostatic Limit of Closed-Shell Interactions Between Two H Atoms. A Critical Review, Hydrogen Bonding - New Insights; Grabowski, S. J., Ed.; Springer, Dordrecht, Netherlands, 2006, pp 337-375.

(61) Hernandez-Trujillo, J.; Matta, C. F. Struct. Chem. 2007, 18, 849-857.

(62) Bader, R. F. W. J. Phys. Chem. A 2009, 113, 10391-10396.

(63) Popelier, P. L. A.; Bader, R. F. W. Chem. Phys. Lett. 1992, 189, 542-548.

(64) Koch, U.; Popelier, P. L. A. J. Phys. Chem. 1995, 99, 9747-9754

(65) Popelier, P. L. A. Atoms in Molecules: An Introduction, Prentice Hall, London, 2000.

(66) Luaña, V.; Pendás, A. M.; Costales, A.; Carriedo, G. A.; García-Alonso, F. J. J. Phys. Chem. A 2001, 105, 5280-5291.

(67) Bader, R. F. W.; Matta, C. F. Inorg. Chem. 2001, 40, 5603-5611.

(68) Varadwaj, P. R.; Cukrowski, I.; Marques, H. M. J Mol Struct (Theochem) 2009, 915, 2032.

(69) King, P.; Clérac, R.; Wernsdorfer, W.; Anson, C. E.; Powell, A. K. Dalton Trans. 2004, 2670-2676. 
Table 1. Computed formation constants, as $\log K_{1}$, of the NiNTPA complex involving lowest energy conformers of ligands ${ }^{46,47}$ and conformers of nickel complexes: self-constructed (S-c) - Test 1; CA1 - Test 2; CA18 of NiNTA and CA4 of NiNTPA - Test 3; CA18 of Ni(NTA) and CA3 of NiNTPA - Test 4.

\begin{tabular}{|c|c|c|c|c|c|c|c|c|}
\hline & \multicolumn{4}{|c|}{$G(\mathbf{a q}) / \mathbf{a u}$} & \multirow[b]{2}{*}{$\log K_{1}$} & \multirow[b]{2}{*}{ Basis set } & \multirow[b]{2}{*}{ Solvation model } & \multirow[b]{2}{*}{$\delta \log K_{1}{ }^{\mathrm{a}}$} \\
\hline & NiNTA & NTPA & NiNTPA & NTA & & & & \\
\hline \multirow[t]{3}{*}{ Test 1} & -2400.031805 & -856.877193 & -2517.911881 & -738.978069 & 2.75 & $6-311+G(d, p)$ & $\mathrm{PCM} / \mathrm{UA} 0$ & -3.05 \\
\hline & -2400.037883 & -856.897797 & -2517.913905 & -739.003179 & 2.96 & $6-311+G(d, p)$ & CPCM/UAKS & -2.84 \\
\hline & -2400.032423 & -856.897237 & -2517.908016 & -739.002625 & 2.76 & $6-311+\mathrm{G}(\mathrm{d}, \mathrm{p})$ & PCM/UAHF & -3.04 \\
\hline Test 2 & -2400.034016 & -856.877193 & -2517.914537 & -738.978069 & 2.95 & $6-311+\mathrm{G}(\mathrm{d}, \mathrm{p})$ & $\mathrm{PCM} / \mathrm{UA} 0$ & -2.85 \\
\hline Test 3 & -2400.038911 & -856.894109 & -2517.914980 & -739.003338 & 4.75 & $6-311++G(d, p)$ & CPCM/UAKS & -1.05 \\
\hline Test 4 & -2400.038911 & -856.894109 & -2517.915860 & -739.003338 & 5.15 & $6-311++G(d, p)$ & CPCM/UAKS & -0.65 \\
\hline
\end{tabular}

a) $\delta \log K_{1}=($ theoretical - experimental) value 
Table2. Non-structural intramolecular interactions in the conformers (NiL) of the NiNTPA complex found from the QTAIM analysis and close contacts 'ignored' by the QTAIM analysis.

Intramolecular bonds

Non-structural rings

CCP

Close contacts

\begin{tabular}{|c|c|c|c|c|c|c|c|c|c|c|c|c|c|c|c|}
\hline NiL & Atoms & $\rho(\mathbf{r})$ & $\nabla^{2} \rho(\mathbf{r})$ & $\AA$ & Atoms & & $\rho(\mathbf{r})$ & & & $\nabla^{2} \rho(\mathbf{r})$ & & $\rho(\mathbf{r})$ & $\nabla^{2} \rho(\mathbf{r})$ & Atoms & $\AA ̊$ \\
\hline \multirow[t]{4}{*}{ CA4 } & & & & & $\mathrm{N}-\mathrm{C} 2-\mathrm{C} 3-\mathrm{H} 26-\mathrm{H} 29-\mathrm{C} 6-\mathrm{C} 5$ & 0.0082 & \multirow{3}{*}{$\begin{array}{l}\text { Aver }^{\mathbf{a}} \\
0.0074\end{array}$} & \multirow{3}{*}{$\begin{array}{c}\text { StDev }^{\mathbf{b}} \\
0.0015\end{array}$} & 0.0371 & \multirow{3}{*}{$\begin{array}{l}\text { Aver }^{\mathrm{a}} \\
0.0306\end{array}$} & \multirow{3}{*}{$\begin{array}{l}\text { Stdev }^{\mathbf{b}} \\
0.0075\end{array}$} & \multirow{3}{*}{0.0051} & \multirow[b]{2}{*}{0.0230} & $\mathrm{OH} 21-\mathrm{O} 11 \mathrm{C}$ & \multirow{2}{*}{$\begin{array}{l}2.523 \\
2.445\end{array}$} \\
\hline & $\mathrm{CH} 26-\mathrm{H} 29 \mathrm{C}$ & 0.0106 & 0.0362 & 2.136 & Ni-O13-C7-C6-H29-H26-C3-C4-O11 & 0.0057 & & & 0.0223 & & & & & $\mathrm{OH} 22-\mathrm{O} 15 \mathrm{C}$ & \\
\hline & $\mathrm{H} 28-\mathrm{O} 17 \mathrm{H}$ & 0.0093 & 0.0293 & 2.583 & Ni-N-C5-H28-O17 & 0.0082 & & & 0.0322 & & & & & & \\
\hline & $\mathrm{CH} 24-\mathrm{H} 34 \mathrm{C}$ & 0.0139 & 0.0490 & 1.964 & N-C2-H24-H34-C9-C8 & 0.0133 & & & 0.0595 & & & & & & \\
\hline \multirow[t]{4}{*}{ CA6 } & GUר & (200 & & & N-C2-C3-H26-H29-C6-C5 & 0.0082 & \multirow{3}{*}{$\begin{array}{l}\text { Aver }^{\mathbf{a}} \\
0.0073\end{array}$} & \multirow{3}{*}{$\begin{array}{l}\text { StDev }^{\mathbf{b}} \\
0.0014\end{array}$} & 0.0367 & \multirow{3}{*}{$\begin{array}{l}\text { Aver }^{\mathbf{a}} \\
0.0299\end{array}$} & \multirow{3}{*}{$\begin{array}{r}\text { StDev }^{\mathbf{b}} \\
0.0073\end{array}$} & \multirow{3}{*}{0.0052} & \multirow[b]{2}{*}{0.0232} & $\mathrm{OH} 21-\mathrm{O} 13 \mathrm{C}$ & \multirow{2}{*}{$\begin{array}{l}2.607 \\
2.575\end{array}$} \\
\hline & СН26-H29C & 0.0102 & 0.0353 & 2.150 & Ni-O13-C7-C6-H29-H26-C3-C4-O11 & 0.0057 & & & 0.0223 & & & & & $\mathrm{OH} 22-\mathrm{O} 11 \mathrm{C}$ & \\
\hline & $\mathrm{CH} 28-\mathrm{O} 17 \mathrm{H}$ & 0.0088 & 0.0284 & 2.563 & Ni-N-C5-H28-O17 & 0.0080 & & & 0.0307 & & & & & & \\
\hline & $\mathrm{CH} 24-\mathrm{H} 34 \mathrm{C}$ & 0.0140 & 0.0498 & 1.963 & N-C2-H24-H34-C9-C8 & 0.0135 & & & 0.0603 & & & & & & \\
\hline \multirow[t]{4}{*}{ S-c } & $\mathrm{CH} 22017 \mathrm{C}$ & 00002 & & & Ni-O17-H33-C9-C10-O15 & 0.0066 & \multirow{3}{*}{$\begin{array}{l}\text { Aver }^{\mathbf{a}} \\
0.0071\end{array}$} & \multirow{3}{*}{$\begin{array}{r}\text { StDev }^{\mathbf{b}} \\
0.0016\end{array}$} & 0.0290 & \multirow{3}{*}{$\begin{array}{l}\text { Aver }^{\mathbf{a}} \\
0.0296\end{array}$} & \multirow{3}{*}{$\begin{array}{r}\text { StDev }^{\mathbf{b}} \\
0.0091\end{array}$} & \multirow{3}{*}{0.0054} & \multirow{3}{*}{0.0251} & CH28-H33C & \multirow{3}{*}{$\begin{array}{l}2.200 \\
2.108\end{array}$} \\
\hline & CH33-OI/C & 0.0092 & 0.0320 & 2.520 & N-C5-H28-O17-H33-C9-C8 & 0.0061 & & & 0.0235 & & & & & CH24-H29C & \\
\hline & $\mathrm{CH} 28-\mathrm{O} 17 \mathrm{H}$ & 0.0111 & 0.0386 & 2.438 & Ni-N-C5-H28-O17 & 0.0084 & & & 0.0364 & & & & & & \\
\hline & $\mathrm{CH} 25-\mathrm{H} 32 \mathrm{C}$ & 0.0144 & 0.0506 & 1.944 & N-C2-C3-H25-H32-C8 & 0.0136 & & & 0.0621 & & & & & & \\
\hline \multirow[t]{4}{*}{ CA1 } & & & & & N-C2-C3-H25-H34-C9-C8 & 0.0081 & \multirow{3}{*}{$\begin{array}{l}\text { Aver }^{\mathbf{a}} \\
0.0077\end{array}$} & \multirow{3}{*}{$\begin{array}{r}\text { StDev }^{\mathbf{b}} \\
0.0023\end{array}$} & 0.0362 & \multirow{3}{*}{$\begin{array}{l}\text { Aver }^{\mathbf{a}} \\
0.0331\end{array}$} & \multirow{3}{*}{$\begin{array}{r}\text { StDev }^{\mathbf{b}} \\
0.0114\end{array}$} & \multirow{3}{*}{0.0053} & & $\mathrm{OH} 22-\mathrm{O} 15 \mathrm{C}$ & 2.690 \\
\hline & $\mathrm{CH} 25-\mathrm{H} 34 \mathrm{C}$ & 0.0098 & 0.0343 & 2.206 & C3-C4-O11-Ni-O15-C10-H34-H25 & 0.0059 & & & 0.0235 & & & & 0.0240 & $\mathrm{OH} 21-\mathrm{O} 13 \mathrm{C}$ & 2.719 \\
\hline & CH31-O17H & 0.0119 & 0.0416 & 2.392 & Ni-N-C8-H31-O17 & 0.0092 & & & 0.0397 & & & & & & \\
\hline & $\mathrm{CH} 24-\mathrm{H} 29 \mathrm{C}$ & 0.0138 & 0.0491 & 1.971 & N-C2-H24-H29-C6-C5 & 0.0134 & & & 0.0596 & & & & & & \\
\hline CA3 & & & & & N-C2-C3-H25-H34-C9-C8 & 0.0082 & & & 0.0367 & & & & & $\mathrm{OH} 22-\mathrm{O} 11 \mathrm{C}$ & 2.452 \\
\hline & $\mathrm{CH} 25-\mathrm{H} 34 \mathrm{C}$ & 0.0102 & 0.0351 & 2.169 & C3-C4-O11-Ni-O15-C10-H34-H25 & 0.0060 & $\begin{array}{l}\text { Aver }^{\mathrm{a}} \\
0.0073\end{array}$ & $\begin{array}{l}\text { StDev }^{\mathbf{b}} \\
0.0014\end{array}$ & 0.0237 & $\begin{array}{l}\text { Aver }^{\mathrm{a}} \\
0.0302\end{array}$ & $\begin{array}{l}\text { StDev }^{\mathbf{b}} \\
0.0046\end{array}$ & 0.0053 & 0.0240 & $\mathrm{OH} 21-\mathrm{O} 13 \mathrm{C}$ & 2.533 \\
\hline & CH31-O17C & 0.0086 & 0.0270 & 2.629 & Ni-N-C8-H31-O17 & 0.0079 & & & 0.0302 & & & & & & \\
\hline & $\mathrm{CH} 24-\mathrm{H} 29 \mathrm{C}$ & 0.0139 & 0.0493 & 1.965 & N-C2-H24-H29-C6-C5 & 0.0134 & & & 0.0596 & & & & & & \\
\hline & & & & & & & & & & & Average: & 0.0053 & 0.0239 & & \\
\hline & & & & & & & & & & & StDev: & 0.0001 & 0.0010 & & \\
\hline
\end{tabular}

$\left.{ }^{a}\right)$ Aver $=$ average, $^{b}$ ) StDev $=$ standard deviation. 
Table 3. Properties of $\mathrm{H}$-atoms and the stabilization energy, $E_{\text {stab }}$, of $\mathrm{H}-\mathrm{H}$ bonding in the indicated conformers of NiNTPA. $q(\mathrm{H})$ is the charge $(e)$ on an $\mathrm{H}$-atom and $E(\mathrm{H})$ is its atomic energy; $\mathrm{BL}$ is the bond length in the energy-optimized structure. Atoms printed in bold are involved in the $\mathrm{H}-\mathrm{H}$ bonding.

\begin{tabular}{|c|c|c|c|c|c|c|c|}
\hline Conformer & H-H bond & $\begin{array}{c}\text { BLL } \\
\AA\end{array}$ & Atom & $\mathbf{q}(\mathbf{H})$ & $E / \mathbf{a u}$ & $\begin{array}{c}\Delta E \\
{\text { kcal } \text { mol }^{-1}}^{-1}\end{array}$ & $\begin{array}{c}E_{\text {stab }} \\
\text { kcal mol }^{-1}\end{array}$ \\
\hline \multirow{8}{*}{ CA4 } & \multirow{4}{*}{$\mathrm{CH} 26-\mathrm{H} 29 \mathrm{C}$} & \multirow{4}{*}{2.136} & H29 & 0.0317 & -0.61168 & \multirow{2}{*}{-3.09} & \multirow{4}{*}{-7.75} \\
\hline & & & $\mathrm{H} 30$ & 0.0391 & -0.60676 & & \\
\hline & & & H26 & 0.0239 & -0.61964 & \multirow{2}{*}{-4.66} & \\
\hline & & & $\mathrm{H} 25$ & 0.0292 & -0.61221 & & \\
\hline & \multirow{4}{*}{$\mathrm{CH} 24-\mathrm{H} 34 \mathrm{C}$} & \multirow{4}{*}{1.964} & H24 & 0.0297 & -0.62468 & \multirow{2}{*}{-5.71} & \multirow{4}{*}{-5.62} \\
\hline & & & $\mathrm{H} 23$ & 0.0263 & -0.61557 & & \\
\hline & & & H34 & 0.0340 & -0.60850 & \multirow{2}{*}{0.10} & \\
\hline & & & H33 & 0.0364 & -0.60865 & & \\
\hline \multirow{8}{*}{ CA3 } & \multirow{4}{*}{ CH24-H29C } & \multirow{4}{*}{1.965} & H24 & 0.0293 & -0.62522 & \multirow{2}{*}{-6.09} & \multirow{4}{*}{-6.11} \\
\hline & & & $\mathrm{H} 23$ & 0.0264 & -0.61551 & & \\
\hline & & & H29 & 0.0340 & -0.60846 & \multirow{2}{*}{-0.02} & \\
\hline & & & $\mathrm{H} 30$ & 0.0362 & -0.60843 & & \\
\hline & \multirow{4}{*}{$\mathrm{CH} 25-\mathrm{H} 34 \mathrm{C}$} & \multirow{4}{*}{2.169} & $\mathbf{H 2 5}$ & 0.0252 & -0.61915 & \multirow{2}{*}{-4.48} & \multirow{4}{*}{-7.67} \\
\hline & & & $\mathrm{H} 26$ & 0.0295 & -0.61202 & & \\
\hline & & & H34 & 0.0316 & -0.61150 & \multirow{2}{*}{-3.19} & \\
\hline & & & H33 & 0.0397 & -0.60642 & & \\
\hline
\end{tabular}


Table 4. Atomic energies $E(\mathrm{H})$ of $\mathrm{H}$-atoms in the indicated conformers of NiNTPA (atoms in bold are involved in the $\mathrm{H}-\mathrm{H}$ bonding). $\Delta E=E_{\mathrm{H}}($ bonded $)-E_{\mathrm{H}}($ non-bonded $)$ in $\mathrm{kcal} \mathrm{mol}^{-1}$.

\begin{tabular}{llrl}
\hline Conformer & Atom & $\boldsymbol{E}(\mathbf{H}) / \mathbf{a u}$ & $\Delta \boldsymbol{E}$ \\
\hline CA4 & H26 & -0.61964 & -4.78 \\
CA3 & H26 & -0.61202 & \\
CA3 & H25 & -0.61915 & -4.35 \\
CA4 & H25 & -0.61221 & \\
\hline
\end{tabular}


Table 5. Properties of $\mathrm{H}$-atoms forming a $-\mathrm{CH}_{2}-$ fragment involved in the $\mathrm{CH}-\mathrm{O}$ bonding in the indicated conformers of NiNTPA. $q(\mathrm{H})$ is the charge $(e)$ on an $\mathrm{H}$-atom and $E(\mathrm{H})$ is its atomic energy; $\mathrm{BL}$ is the bond length in the energy-optimized structure. Atoms printed in bold are involved in the $\mathrm{CH}-\mathrm{O}$ bonding. $\Delta E=E_{\mathrm{H}}$ (bonded) $-E_{\mathrm{H}}$ (non-bonded).

\begin{tabular}{lcccccc}
\hline \multirow{2}{*}{ Conformer } & \multirow{2}{*}{ Bond } & $\begin{array}{c}\text { BL } \\
\mathbf{A}\end{array}$ & Atom & $\mathbf{q ( H )}$ & $\boldsymbol{E} / \mathbf{a u}$ & $\begin{array}{c}\Delta \boldsymbol{E} \\
\mathbf{k c a l ~ m o l}^{-\mathbf{1}}\end{array}$ \\
\hline \multirow{2}{*}{$\mathrm{CA} 4$} & $\mathrm{C} 5 \mathrm{H}-\mathrm{O} 17$ & 2.583 & $\mathbf{H 2 8}$ & 0.0293 & -0.62313 & -3.33 \\
& & & $\mathrm{H} 27$ & 0.0177 & -0.61782 & \\
\multirow{2}{*}{$\mathrm{CA} 3$} & $\mathrm{C} 8 \mathrm{H}-\mathrm{O} 17$ & 2.629 & $\mathbf{H 3 1}$ & 0.0236 & -0.62562 & -5.13 \\
& & & $\mathrm{H} 32$ & 0.0185 & -0.61744 & \\
\hline
\end{tabular}


Table 6. Atomic energies $E(\mathrm{H})$ of $\mathrm{H}$-atoms in the indicated conformers of NiNTPA (atoms in bold are involved in the $\mathrm{CH}-\mathrm{O}$ bonding). $\Delta E=E_{\mathrm{H}}$ (bonded) $-E_{\mathrm{H}}$ (non-bonded) in $\mathrm{kcal} \mathrm{mol}^{-1}$.

\begin{tabular}{llrl}
\hline Conformer & Atom & $\boldsymbol{E}(\mathbf{H}) / \mathbf{a u}$ & $\Delta \boldsymbol{E}$ \\
\hline CA4 & H28 & -0.62313 & -2.56 \\
CA3 & H28 & -0.61906 & \\
CA3 & H31 & -0.62562 & -4.25 \\
CA4 & H31 & -0.61885 & \\
\hline
\end{tabular}


Table 7. The strain energy, $E_{\mathrm{s}}$, in the as-in-complex ligands (NTA and NTPA) in the specified conformers of Ni(II) complexes, ML.

\begin{tabular}{|c|c|c|c|c|c|c|c|c|}
\hline \multicolumn{3}{|c|}{ NTPA } & \multicolumn{3}{|c|}{ NTA } & \multirow[b]{2}{*}{$\delta E_{\mathrm{s}}{ }^{\mathbf{b}}$} & \multirow[b]{2}{*}{$E_{\mathrm{s}}$ ratio $^{\mathrm{c}}$} & \multirow[b]{2}{*}{$\log K_{1}$ ratio $^{d}$} \\
\hline ML & $E_{\mathrm{s}}$ & $\log K_{1}^{a}$ & ML & $E_{\mathrm{s}}$ & $\log K_{1}^{a}$ & & & \\
\hline $\mathrm{S}-\mathrm{c}^{\mathrm{e}}$ & 40.00 & \multirow{4}{*}{5.80} & $\mathrm{~S}-\mathrm{c}^{\mathrm{e}}$ & 23.52 & \multirow{4}{*}{11.51} & 16.48 & $167^{\mathrm{e}}$ & \multirow{4}{*}{1.98} \\
\hline $\mathrm{CA} 1^{\mathrm{e}}$ & 40.18 & & $\mathrm{CA} 1^{\mathrm{e}}$ & 24.45 & & 15.73 & \multirow{3}{*}{$1.90^{\mathrm{f}}$} & \\
\hline $\mathrm{CA} 6^{\mathrm{f}}$ & 33.04 & & $\mathrm{CA} 5^{\mathrm{f}}$ & 17.81 & & 15.23 & & \\
\hline $\mathrm{CA} 4^{\mathrm{f}}$ & 34.25 & & CA $18^{f}$ & 17.68 & & 16.57 & & \\
\hline
\end{tabular}

a) Experimental formation constant; $\left.{ }^{2}{ }^{\mathrm{b}}\right) \delta E_{\mathrm{s}}=\frac{1}{\left.\left.E_{\mathrm{s}}(\mathrm{NTPA})-E_{\mathrm{s}}(\mathrm{NTA}),{ }^{\mathrm{c}}\right) E_{\mathrm{s}} \text { ratio }=E_{\mathrm{s}}(\mathrm{NTPA}) / E_{\mathrm{s}}(\mathrm{NTA}),{ }^{\mathrm{d}}\right) \log K_{1} \text { ratio }}=\log K_{\mathrm{NiNTA}} / \log$ $\left.K_{\mathrm{NiNTPA}},{ }^{\mathrm{e}}\right) \mathrm{B} 3 \mathrm{LYP} / 6-311+\mathrm{G}(\mathrm{d}, \mathrm{p})$ using the PCM/UA0 solvation model; $\left.{ }^{f}\right) \mathrm{B} 3 \mathrm{LYP} / 6-311++\mathrm{G}(\mathrm{d}, \mathrm{p})$ using the CPCM/UAKS solvation model. 
